CentroSur

Social Science Journal

\section{Seguridad e higiene industrial en el proceso de producción para la reducción de enfermedades profesionales y accidentes laborales}

Industrial safety and hygiene in the production process for the reduction of professional illnesses and occupational accidents
Centro Sur.

Social Science Journal

Abril 2020 - E3

http://centrosureditorial.com/ind ex.php/revista eISSN: 2600-5743

revistacentrosur@gmail.com Recepción: 4 de marzo 2018 Aprobación 12 junio 2019

Pag 86 - 99

Atribución/ReconocimientoNoComercial-Compartirlgual 4.0 Licencia Pública Internacional CC BY-NC-SA 4.0 https://creativecommons.org/lice nses/by-nc-sa/4.0/legalcode.es

\author{
Pérez Toapanta Adriano ${ }^{1}$ \\ Aguilera Daza Fabricio Raúl ${ }^{2}$ \\ Cañizares Arcila Andrés Eduardo ${ }^{3}$ \\ Erazo Jiménez Geolene Marbel ${ }^{4}$
}

\section{Resumen}

El presente proyecto se llevó a cabo a la realización de un plan de seguridad industrial e higiene del proceso de producción en la empresa INDUHORST, para la reducción de enfermedades profesionales $y$ accidentes laborales para la comercialización de equipos que se diseñan y se producen en las instalaciones de la empresa INDUHORST. Intervienen 6 procesos principales para la producción de maquinarias; cortado, doblado, soldado, perforado, torneado y pintado. A través de la matriz de GTC-45 se detectó los riesgos y peligros a los que están expuestos los trabajadores al realizar cada tarea al momento de desempeñar sus actividades laborales. Para ello se hizo una propuesta de una alternativa de solución que reduzca las enfermedades y accidentes laborales en la empresa, pero para establecer dicha solución se realizaron estudios directamente en el proceso de producción, con los trabajadores y las máquinas para obtener valores y poder evaluar cada riesgo y peligro existente. A través de la identificación de riesgos se determinaron varios riesgos hacia los trabajadores, tales como: Ruido, Mala postura, Caídas, Ergonómico y Movimientos repetitivos.

\footnotetext{
${ }^{1}$ Magister en Seguridad e Higiene Industrial y Ambiental. Adriano Efraín Pérez Toapanta. Quevedo.Quevedo,Ecuador. ORCID:0000-0003-38907313. Email; aeperez@uteq.edu.ec

2 Estudiante. Fabricio Raúl Aguilera Daza. Quevedo. Quevedo, Ecuador. ORCID: 0000-0002-4002-1311. Email: fabricio.aguilera2016@uteq.edu.ec

${ }^{3}$ Estudiante. Andrés Eduardo Cañizares Arcila. Quevedo. Quevedo, Ecuador. ORCID: 0000-0002-1621-8229. Email; andres.canizares2016@uteq.edu.ec

4Estudiante. Geolene Marbel Erazo Jiménez. Quevedo. Quevedo, Ecuador. ORCID: 0000-0003-2227-7687. Email;geolene.erazo2016@uteq.edu.ec
} 
Palabras clave: ergonomía, riesgo, seguridad

\section{ABSTRACT}

The present project will carry out an industrial safety and hygiene plan for the production process in the company INDUHORST, for the reduction of professional illnesses and occupational accidents for the marketing of equipment that is designed and produced in the installations. of the company INDUHORST. Intervene 6 main processes for the production of machinery; cut, folded, welded, perforated, turned and painted. Through the GTC-45 matrix it is possible to detect the risks and dangers to the workers who are carrying out each task at the moment of carrying out their labor activities. For him there is a proposal for an alternative solution that reduces them sicknesses and accidents at work in the company, but to establish a solution, studies were carried out directly in the production process, with workers and machines to obtain values and be able to evaluate each existing risk and danger. Through the identification of strains, several strains have been determined for the workers, such as: Noise, Suitcase, Falling, Ergonomic and repetitive movements.

Keywords: ergonomics, risk, safety

\section{Introducción}

En la actualidad toda industria o empresa que desean ser competitivas en el mercado deberán tener su plan de seguridad e higiene laboral, trabajando en equipo con recursos humanos, de tal manera presentaran resultados favorables para las personas que trabajan en ella y a su vez los gerentes tienen la obligación de brindar seguridad a sus trabajadores.

Este proyecto se desarrolla en la empresa INDUHORST CÍA. LTDA. se dedica a la comercialización de equipos y repuestos que se diseñan y producen en las instalaciones de las productoras, los materiales utilizados son de primera calidad, bajo marcas de reconocido prestigio mundial.

La falta de un plan de seguridad a afecta a la empresa debido a que existe mucha competencia tanto a nivel nacional e internacional, las empresas están obligadas a mejorar la seguridad y a la salud de sus empleados mediante la prevención de riesgos laborales, evitando de esta manera que se produzcan accidentes laborales y enfermedades profesionales que puedan afectar a la vida de los trabajadores y generar costos económicos.

Para la realización del plan de seguridad se tienen que poner en práctica las medidas de seguridad basadas en la evaluación de riesgos y en la legislación pertinente 
además de factores que se deben tomar en cuenta al momento de realizar todo el proceso, lo importante será la reducción de accidentes e incidentes que se presenten lo cual será de gran beneficio para el trabajador.

El presente trabajo se lo va a realizar en la empresa INDUHORST CIA. LDA. con el fin de proponer un plan de seguridad Industrial para reducir enfermedades profesionales y accidentes laborales mediante un análisis de los puestos de trabajo para lograr evitar posibles accidentes que se pueden dar debido a que no se utiliza la maquinaria, el material, las herramientas, o el equipo de protección adecuado debido a la falta de estudio o de información como son las capacitaciones hacia los trabajadores.

El estudio que se va a realizar será mediante la realización de una matriz de prevención de riegos con el fin de identificar factores de riegos existentes y actuar minuciosamente y lograr eliminar estos posibles riesgos o minimizar los mismos para salvaguardar la seguridad y salud del trabajador tomando medidas preventivas logrando solucionar este problema que afecta directamente a los trabajadores por no tomar las medidas de seguridad adecuadas.

Las empresas que no cuentan con un plan de seguridad industrial, tienden a ser afectas en su rendimiento y producción, bajando considerablemente la eficiencia y la eficacia del trabajador dando como tal una baja productividad a la empresa.

Las máquinas y herramientas que no cuentan con las condiciones de seguridad y salud para la utilización de los trabajadores pueden ocasionar lesiones por aplastamiento, cizallamiento, corte, enganche, atrapamiento, impacto, punzonamiento, fricción-abrasión, proyección de fluido, choque eléctrico, cortocircuitos, sordera u otros daños, debido a no ser máquinas ergonómicamente diseñadas con una evaluación de riesgo que sea aceptable para el trabajador o por la incorrecta utilización de las herramientas, equipos y máquinas.

La mano de obra debe ser especializada, al momento de no contar con personal especializado y capacitado para la realización de sus labores, este puede omitir algunas obligaciones como la de utilizar los equipos de protección personal, realizar posturas forzadas, movimientos repetitivos, mala manipulación de carga que generaran lesiones en su cuerpo o provocar una enfermedad profesional.

Cuando un trabajador no cumple con el reglamento de seguridad laboral de la empresa, genera un daño para sí mismo provocando accidentes o enfermedades profesionales debido a la falta de conocimiento.

Si los materiales que se utiliza para la elaboración de un producto no se encuentran en buen estado, y tienen un peso que sobrepasa al peso recomendable, esto provoca lesiones dorso lumbares distensiones o roturas musculares, contusiones, heridas y/o cortes. 
Si el medio en el cual se encuentran los trabajadores, esta desordenado y con una limpieza deficiente provoca caídas y de esta manera lesiones en el trabajador.

La seguridad industrial es una realidad compleja, que abarca desde problemática estrictamente técnica hasta diversos tipos de efectos humanos y sociales. A la vez, debe ser una disciplina de estudio en la que se han de formar los especialistas apropiados, aunque su naturaleza no corresponde a las asignaturas académicas clásicas, sino a un tipo de disciplina de corte profesional, aplicado y con interrelaciones legales muy significativas.

INDUHORST Cía. Ltda. Se dedica a la comercialización de equipos y repuestos que se diseñan y producen en las instalaciones de las productoras, los materiales utilizados son de primera calidad, bajo marcas de reconocido prestigio mundial. Cuenta con más de 1500 clientes entre distribuidores y usuarios finales. Somos una de las primeras empresas en prestigio a nivel nacional. Este logro y todos los esfuerzos presentes y futuros están inspirados en un solo objetivo: el mayor beneficio para el cliente.

El desarrollo de este proyecto aporta al proceso formativo como Ingenieros Industriales, aplicando el aprendizaje en curso al entorno laboral, mediante la identificación de riesgos a los que están expuestos los operarios en las actividades que desempeñan, por consiguiente, plantear una propuesta con políticas internas acorde para la empresa en función de seguridad industrial, que de ser implementado ofrece un ambiente laboral de bienestar, eficiencia y productividad.

La seguridad industrial en el concepto moderno significa más que una simple situación de seguridad física, una situación de bienestar personal, un ambiente de trabajo idóneo, una economía de costos importantes y una imagen de modernización y filosofía de vida humana en el marco de la actividad laboral contemporánea. (Cavassa, 2005)

La sociedad industrial hace poco dio preferencia a la máquina, el tiempo y el movimiento buscando la maximización de beneficios, sin tomar en cuenta al hombre, elemento básico de todo el engranaje productivo. La política de personal, como toda política, cambia su estrategia, y progresista. Así, el objetivo común es el bienestar del hombre mediante un esfuerzo racionalizado y humanizado, de flexibilidad y seguridad. El trabajo taylorizado se preocupó del rendimiento humano, tratando al individuo como máquina y explotando al máximo sus energías, sin considerarlo como ser humano y pensante. (Cavassa, 2005)

La organización científica del trabajo mide el rendimiento del trabajador, cronometra sus tiempos y concede primas al que más rinde. Con este criterio consigue un aumento aparente de la producción y que el hombre se sienta menos realizado. El ritmo del trabajo está determinado por la máquina de la que el individuo es su esclavo. 
La seguridad de empleo es incierta, los continuos remplazos por ausentismos y rotación de puesto aumenta en forma indirecta la predisposición a los accidentes y sus causas, lo que crea falta de seguridad en el trabajo. (Cavassa, 2005)

Jardiller, en su obra L' Organization Humaine du Travail, menciona como una de las consecuencias negativas de la organización científica el ausentismo profesional debido, en algunos casos, a las enfermedades de trabajo. (Cavassa, 2005)

En Italia, el ausentismo por enfermedades pasó de 5\% al 15\% en 1972.

En Alemania, casi se duplicó en 15 años pasando de 4\% en 1954 a 7.4\% en 1971.

En las plantas Ford pasó de 2.8\% en 1960 a 5.3\% en 1970.

En Fiat, durante el mismo periodo pasó de 6\% a 11\%. (Cavassa, 2005)

El desarrollo Industrial trajo el incremento de accidentes laborales, lo que obligó a aumentar las medidas de seguridad, las cuales se cristalizaron con el advenimiento de las conquistas laborales. (Cavassa, 2005)

Desde los albores de la historia, el hombre ha hecho de su instinto de conservación una plataforma de defensa ante la lesión corporal, tal esfuerzo probablemente fue en principio de carácter personal, instintivo defensivo. Así nació la Seguridad Industrial, reflejada en un simple esfuerzo individual más que en un sistema organizado. (Cavassa, 2005)

Ya en el año 400 A.C. Hipócrates recomendaba a los mineros el uso de baños higiénicos a fin de evitar la saturación del plomo. También Platón y Aristóteles estudiaron ciertas deformaciones físicas producidas por ciertas actividades ocupacionales, planteando la necesidad de su prevención. Con la Revolución Francesa se establecen corporaciones de seguridad destinadas a resguardar a los artesanos, base económica de la época. (Cavassa, 2005)

La Revolución Industrial marca el inicio de la Seguridad Industrial como consecuencia de la aparición de la fuerza del vapor y la mecanización de la industria, lo que produjo el incremento de accidentes y enfermedades laborales. No obstante, el nacimiento de la fuerza industrial y el de la seguridad industrial no fueron simultáneos, debido a la degradación y a las condiciones de trabajo y de vida detestables. Es decir, en 1871 el cincuenta por ciento de los trabajadores moría antes de los veinte años, debido a los accidentes y las pésimas condiciones de trabajo. (Cavassa, 2005)

En 1833 se realizaron las primeras inspecciones gubernamentales, pero hasta 1850 se verificaron ciertas mejoras como resultado de las recomendaciones hechas entonces. Se acortó la jornada laboral, se estableció un mínimo de edad para los niños trabajadores y se vio algunas mejoras en las condiciones de seguridad. No obstante, los legisladores tardaron demasiado en legislar sobre el bien común del trabajador, pues los conceptos sobre el valor humano y la capitalización del esfuerzo laboral no tenían sentido frente al lucro indiscriminado de los empresarios. Sin embargo, suma a 
su haber el desconocimiento de ciertas técnicas y adelantos que estaban en desarrollo, con los cuales se habrían evitado muchos accidentes y enfermedades laborales. (Cavassa, 2005)

En 1833 se coloca la primera piedra de la seguridad industrial moderna, cuando en París se establece una empresa que asesora a los industriales. Pero es hasta este siglo que el tema de la seguridad en el trabajo alcanza su máxima expresión al crearse la Asociación Internacional de Protección de los Trabajadores. En la actualidad la Oficina Internacional del Trabajo OIT, constituye el organismo rector y guardián de los principios e inquietudes referentes a la seguridad del trabajador en todos los aspectos. (Cavassa, 2005)

Según el ámbito o la época, en lugar del término seguridad laboral se utilizan o se han utilizado diversas denominaciones para un concepto que en realidad es único: las condiciones bajo las que se trabaja deben ser seguras, es decir, no deben suponer una amenaza o una posibilidad significativa de sufrir un daño de cierta entidad, que pueda incapacitar aunque sea parcial y temporalmente, por parte de los trabajadores en relación con el trabajo. (Ríos, 2002)

Se trata, por lo tanto, de un asunto estrictamente laboral, con lo que son los trabajadores quienes deben estar suficientemente protegidos para que la posibilidad de sufrir un daño con ocasión del trabajo sea mínima. Puesto que los trabajadores por cuenta ajena desempeñan las tareas asignadas por el empresario bajo su dirección y en las condiciones de trabajo impuestas por él, es éste quien debe garantizar que tales tareas se lleven a cabo con el menor riesgo de sufrir un daño posible. (Ríos, 2002)

Si bien la protección de la seguridad y la salud de los trabajadores en el trabajo, a través fundamentalmente de la acción preventiva en los centros de trabajo, refleja un estado cultural de fines del siglo $X X$, no deja de ser una consecuencia más del desarrollo de la humanidad y de su incesante evolución a través de la Historia. Evolución no solo de índole cultural, sino también técnica, científica, sociológica y económica. (Ríos, 2002)

En el ámbito de la seguridad laboral, la referencia a las condiciones de trabajo se efectúa con la consideración de que el empresario debe controlar tales condiciones para que no supongan una amenaza para la seguridad y la salud del trabajador $\mathrm{y}$, al mismo tiempo, se alcance una calidad de trabajo. En este sentido, se trata de aquellas características del trabajo que pueden influir significativamente en la generación de riesgos laborales. Se incluye en ellas: (Ríos, 2002)

Condiciones de seguridad

Características generales de los locales (espacios, pasillos, suelos, escaleras, etc.) Instalaciones (eléctrica, de gases, de vapor, etc.) 
Equipos de trabajo (máquinas, herramientas, aparatos a presión, de elevación, de manutención, etc.)

Almacenamiento y manipulación de cargas $u$ otros objetos, de materiales y de productos.

Existencia o utilización de materiales o productos inflamables.

Existencia o utilización de productos químicos peligrosos en general. -Condiciones ambientales:

Exposición a agentes físicos (ruido, vibraciones, radiaciones ionizantes, radiación ultravioleta, radiación infrarroja, microondas, ondas de radio, láser, campos electromagnéticos...)

Exposición a agentes químicos y ventilación industrial.

Exposición a agentes biológicos.

Calor y frío.

Climatización y ventilación general. Calidad del aire.

Iluminación. Carga de trabajo: física y mental. Organización y ordenación del trabajo (monotonía, repetitividad, posibilidad de iniciativa, aislamiento, participación, tonicidad, descansos...) (Ríos, 2002)

En la medida en que estas condiciones de trabajo puedan ser origen de daños para la salud, incluidas las lesiones (es decir, accidentes, patologías o enfermedades), o influyan significativamente en la magnitud de los riesgos, se las suele denominar factores de riesgo o también peligros, situaciones, actividades, condiciones, peligrosas, o como dice la Ley de Prevención: procesos, actividades, operaciones, equipos o productos potencialmente peligrosos (Ríos, 2002)

Esto significa que la seguridad laboral debe mejorar, y en ello todos somos responsables: gobierno, empresa, sindicatos y trabajadores. Una medida clave para conseguir esta mejora a la que hacemos referencia es la formación del trabajador. (Ríos, 2002)

Esta formación debe ser específica en el puesto de trabajo y relativa a prevención de riesgos laborales. El trabajador debe conocer cuáles son sus riesgos y cómo poder evitarlos, y en esto juega un papel esencial la información que se le trasmita desde la dirección de la empresa. (Ríos, 2002)

\section{Materiales y métodos}

Mediante este tipo de investigación se reflejó los datos e información más relevantes detallando los procedimientos y características actuales comprendidas en libros y otros tipos documentos basados en seguridad industrial para la elaboración de un plan de seguridad. Este tipo de investigación fue necesaria para la elaboración del plan de seguridad y el proyecto en su totalidad, puesto que se utilizó investigaciones sencillas 
como, libros, páginas web, con el fin de aplicar la información. La aplicación de este tipo de investigación se empleó para la obtención de información precisa porque se acudió a la empresa a recolectar información real aplicando entrevista al personal involucrado en el caso de estudio. Se presenció los aspectos al cual están sometidos los operarios en sus labores y posteriormente especificar los aspectos o riesgos a evaluar. El método deductivo conllevo al análisis de información colectiva hasta lo individual acerca de los diferentes factores de riesgo al que se exponen los operarios, mediante el uso de herramientas informáticas que evaluaron los puestos de trabajo en el área de producción. Analizó la información de los riesgos evaluados desde forma individual a colectiva, siendo el soporte los datos y mediciones recolectados en el campo de estudio.

\section{Fuente primaria}

Por medio de la observación y entrevista directa con el Jefe de Producción y sus trabajadores se obtuvo información acerca del plan de seguridad e higiene laboral en la empresa INDUHORST CIA. LDA. para realizar la investigación en el área de producción.

\section{Fuente secundaria}

La información se obtuvo mediante libros, páginas web y sitios relacionados con la seguridad e higiene laboral.

\section{Resultados}

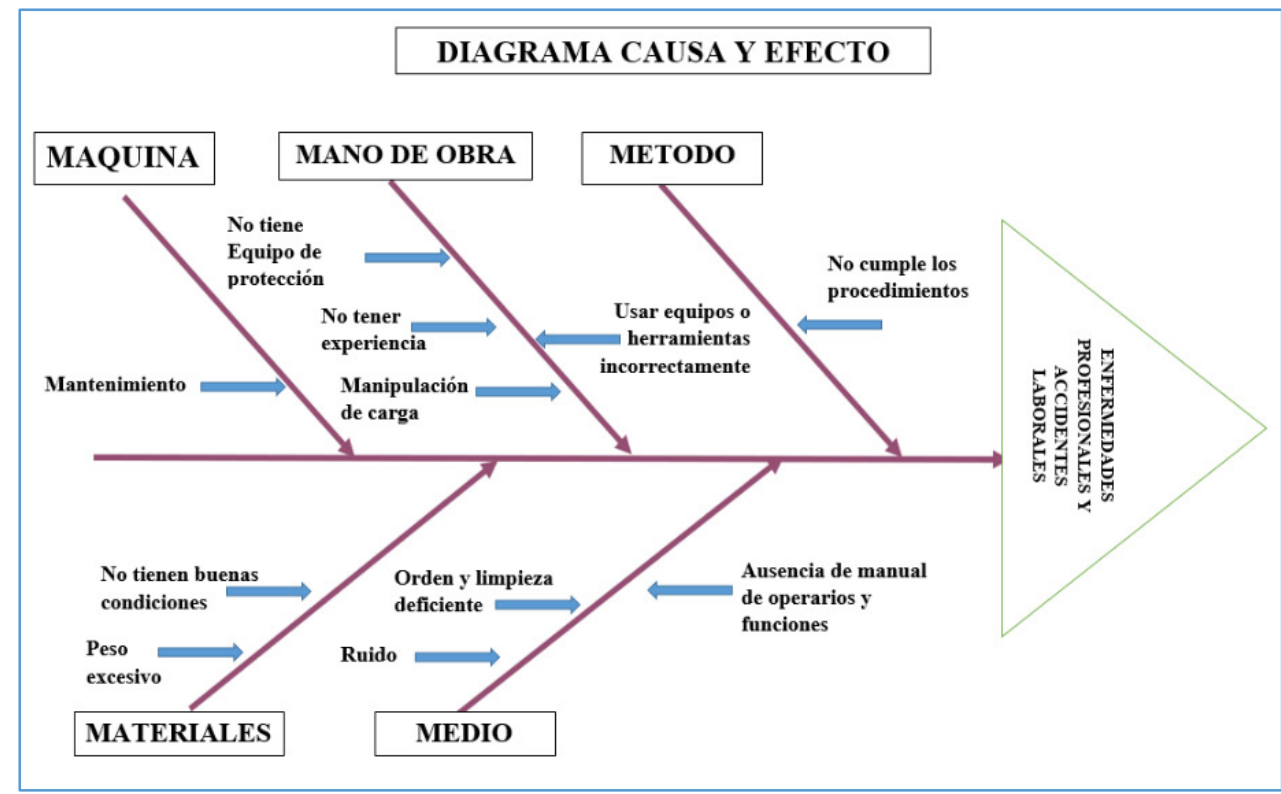

Las empresas que no cuentan con un plan de seguridad industrial, tienden a ser afectas en su rendimiento y producción, bajando considerablemente la eficiencia y la eficacia del trabajador dando como tal una baja productividad a la empresa. 
Mediante el estudio de la matriz GTC45 se observó que en el área de producción en el proceso de elaboración de la máquina envejecedora de arroz, los trabajadores que participan en estas actividades están expuestos a diferentes tipos de riesgo.

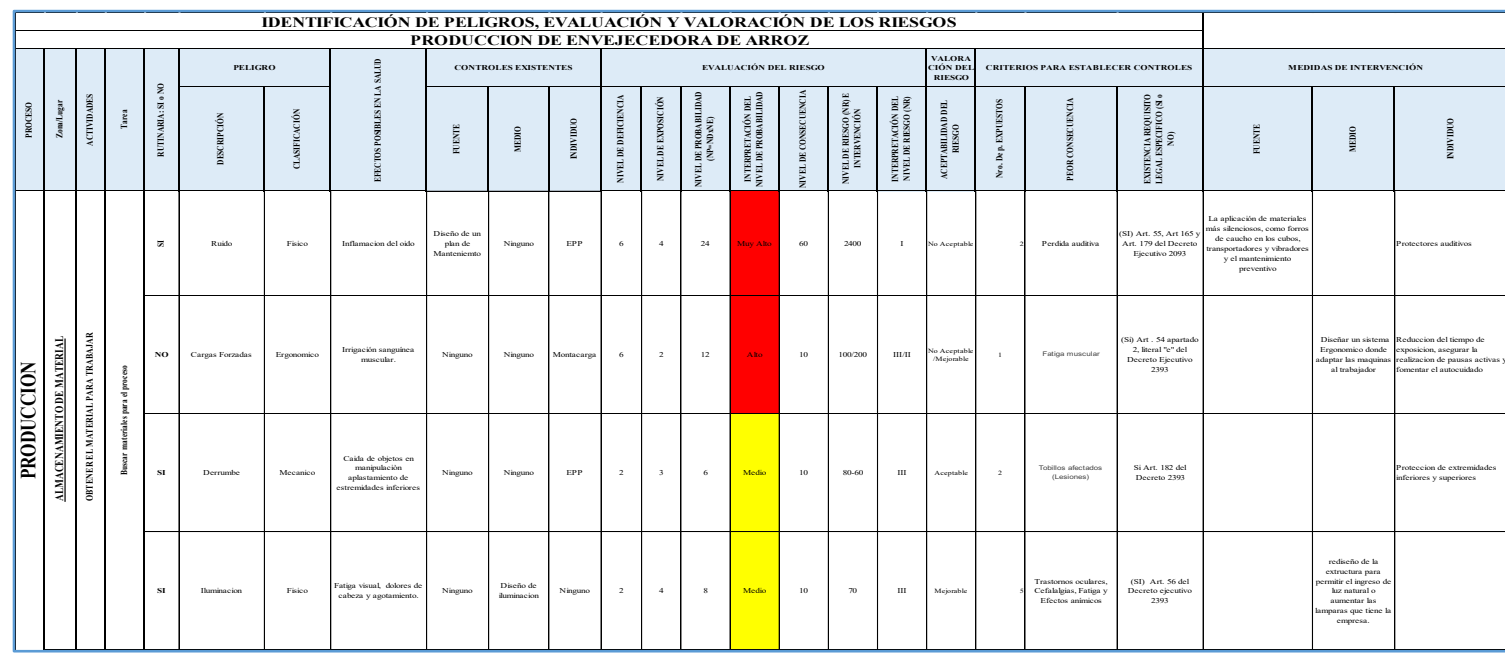

$\begin{array}{lll}\text { CLASIFICACIÓN } & & \\ \text { Físicos } & 9 & 26 \% \\ \text { Ergonómicos } & 5 & 14 \% \\ \text { Mecánicos } & 13 & 37 \% \\ \text { Químicos } & 7 & 20 \% \\ \text { Fuego y explosión } & 1 & 3 \% \\ \text { TOTAL } & \mathbf{3 5} & \mathbf{1 0 0} \%\end{array}$

Según los resultados obtenidos debido a los cálculos realizados a cada una de las áreas de producción se determinó que en el proceso de Pre ensamble, corte, ensamble y doblado sobrepasan el nivel de decibeles según lo establecido en el Reglamento, Salud y Seguridad de los Trabajadores y mejoramiento del medio ambiente de trabajo, Decreto Ejecutivo 2393 donde indica que al laborar durante 8 horas el nivel de decibeles debe ser 85 y al sobrepasar estos decibeles se debe trabajar en la misma situación por un periodo de tiempo más corto. 


\begin{tabular}{|c|c|c|c|c|c|c|c|c|c|c|}
\hline ÁREA & DB & $\begin{array}{l}\text { Promedio } \\
\text { DB }\end{array}$ & $\begin{array}{l}\text { Tiempo } \\
\text { real }\end{array}$ & $\begin{array}{l}\text { Tiempo } \\
\text { permitido }\end{array}$ & Dosis & $\begin{array}{l}\text { (PromdB } \\
-\mathrm{dB})^{2}\end{array}$ & $\sum_{(\text {PromdB-dB })} 2$ & $\mu$ & $\begin{array}{l}\mho \\
=\mu * 2\end{array}$ & Incertidumbre \\
\hline Corte & $\begin{array}{c}86 \\
90 \\
92,8 \\
93 \\
\end{array}$ & 90,45 & $6 \mathrm{~h}$ & 2,27 & 2,64 & $\begin{array}{c}19,80 \\
0,20 \\
5,52 \\
6,50 \\
\end{array}$ & 32,02 & 1,63 & 3,26 & {$[93,71 ; 87,19] \mathrm{dB}$} \\
\hline Doblado & $\begin{array}{l}87 \\
86 \\
85 \\
88 \\
\end{array}$ & 86,5 & $7 \mathrm{~h}$ & 5,66 & 1,24 & $\begin{array}{l}0,25 \\
0,25 \\
2,25 \\
2,25 \\
\end{array}$ & 5 & 0,65 & 1,3 & {$[87,8 ; 85,2] \mathrm{dB}$} \\
\hline $\begin{array}{c}\text { Pre } \\
\text { ensamble }\end{array}$ & $\begin{array}{c}103 \\
106 \\
102 \\
101,5 \\
\end{array}$ & 103,13 & $8 \mathrm{~h}$ & 0,12 & 66,67 & $\begin{array}{l}0,02 \\
8,24 \\
1,28 \\
2,66 \\
\end{array}$ & 12,2 & 1,01 & 2,02 & {$[105,15 ; 101,11] \mathrm{dB}$} \\
\hline Ensamble & $\begin{array}{c}95 \\
98,4 \\
96,6 \\
97\end{array}$ & 96,75 & $8 \mathrm{~h}$ & 0,53 & 15,09 & $\begin{array}{l}3,06 \\
2,72 \\
0,02 \\
0,06\end{array}$ & 5,86 & 0,70 & 1,4 & {$[98,15 ; 95,35] \mathrm{dB}$} \\
\hline Acabados & $\begin{array}{c}79,7 \\
82,5 \\
83,20 \\
81,6\end{array}$ & 81,75 & $7 \mathrm{~h}$ & 8 & 0,88 & $\begin{array}{l}4,20 \\
0,56 \\
2,10 \\
0,02\end{array}$ & 6,88 & 0,76 & 1,52 & {$[83,27 ; 80,23] \mathrm{dB}$} \\
\hline
\end{tabular}

Conforme los resultados obtenidos al cálculo de luminarias en el área de producción se verifico que, en el proceso de corte y doblado, se obtuvo un valor de 233.83 luxes que es mayor o igual que 200 luxes, en el área de pre- ensamble y ensamble dio como resultado 28060 luxes que es mayor o igual que 300 luxes, en el área de acabados se obtuvo un resultado de 498.84 luxes que es mayor o igual que 500 luxes. Los niveles de iluminación mínima, según lo establecido en el reglamento, Salud y seguridad de los trabajadores y mejoramiento del medio de trabajo, Decreto ejecutivo 2393, Art. 56. Donde indica los niveles de iluminación mínima para los trabajos específicos y similares.

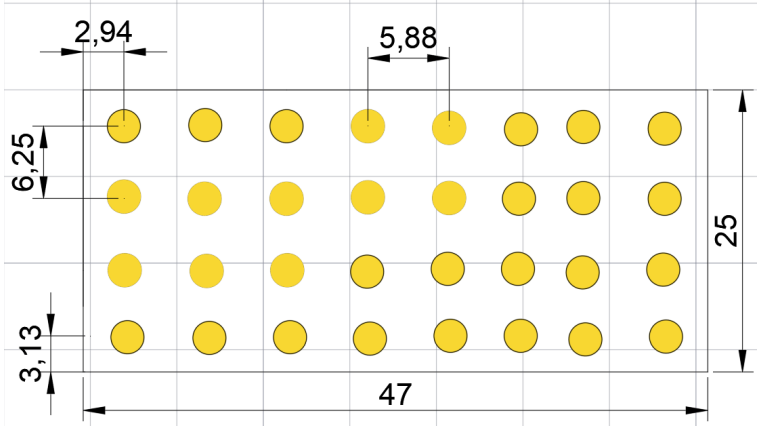

Número de Luminarias Correctas

$$
E m=\frac{N l * n * \phi L * C u * C m}{S}
$$

$E m=\frac{32 * 24 * 1800 \text { lumenes } * 0.53 * 0.8}{1175 \mathrm{~m}^{2}}$

$\geq 500$ luxes

Evaluación de factores de riesgo ergonómicas de posturas forzadas por el método Reba. Las tareas que se van a evaluar a continuación adoptan posturas inadecuadas de forma continua o repetitiva 


\begin{tabular}{|l|c|}
\hline \multicolumn{1}{|c|}{ GRUPO A } & Puntuación \\
\hline Puntuación del tronco & \\
\hline Flexión $>60^{\circ}$ & \\
\hline Puntuación del cuello & 2 \\
\hline Flexión $>20^{\circ}$ o Extensión & \\
\hline Puntuación de las piernas & \\
\hline Sentado, andando o de pie con soporte & \\
\hline Giro de muñeca & \\
\hline Pronación o supinación media & \\
\hline \multicolumn{1}{|c|}{ GRUPO B } & 3 \\
\hline Puntuación del cuello & \\
\hline Flexión $>20^{\circ}$ & 3 \\
\hline Puntuación del tronco & \\
\hline Flexión $>20^{\circ}$ y $\leq 60^{\circ}$ & 3 \\
\hline Puntuación del brazo & \\
\hline Flexión $>45^{\circ}$ y $90^{\circ}$ & 1 \\
\hline Puntuación del antebrazo & \\
\hline Flexión entre 60 $60^{\circ} 100^{\circ}$ & 2 \\
\hline Puntuación de la muñeca & \\
\hline Flexión o extensión $>15^{\circ}$ & \\
\hline
\end{tabular}

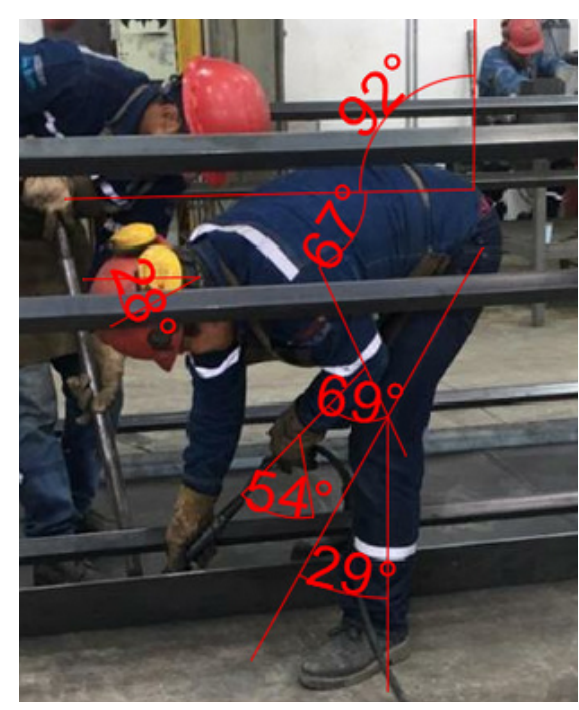

La puntuación final es 7 siendo un nivel de riesgo medio y la actuación es necesaria.

Planteamiento de alternativas de solución que reduzca las enfermedades profesionales y accidentes laborales en la empresa Induhorst

- Reglamento de Seguridad y Salud de los Trabajadores y Mejoramiento del Medio.

- Plan de emergencia mediante contingente de incendios $T s=\frac{30 \text { personas }}{2,50 \text { metros } * 1.3 \frac{\text { personas }}{\text { metros } * \text { segundo }}}+\frac{75 \text { metros }}{0.6 \text { metros }}=2.65$ minutos es el tiempo máximo de salida desde el punto más alejado del edificio.

- Procedimientos de trabajo seguro para cada una de las áreas de producción de la empresa

- Controles a los riesgos identificados en el área de producción 


\begin{tabular}{|c|c|c|c|c|}
\hline \multicolumn{5}{|c|}{ CONTROLES } \\
\hline Riesgos & Descripción & Fuente & Medio & Individuo \\
\hline \multirow{4}{*}{ Físicos } & Ruido & $\begin{array}{l}\text { Cambio o } \\
\text { modificación } \\
\text { de procesos, } \\
\text { diseño o } \\
\text { selección de } \\
\text { equipos que } \\
\text { generen } \\
\text { menos ruido. }\end{array}$ & & $\begin{array}{l}\text { Orejeras (Modelo } \\
\text { H10P3E OPTIMETM } \\
105 \text { adosable a casco } \\
\text { y Modelo H10A } \\
\text { OPTIMETM } 105 \text { con } \\
\text { banda superior) } \\
\text { Desarrollado para una } \\
\text { protección completa } \\
\text { en los entornos de } \\
\text { ruidos más exigentes. }\end{array}$ \\
\hline & |lluminación & \begin{tabular}{|l} 
Tener un \\
programa de \\
mantenimiento \\
preventivo y \\
limpieza a las \\
luminarias.
\end{tabular} & $\begin{array}{l}\text { Tener una buena } \\
\text { distribución de las } \\
\text { luminarias }\end{array}$ & \\
\hline & $\begin{array}{l}\text { Exposición a } \\
\text { temperaturas }\end{array}$ & & $\begin{array}{l}\text { Sistema de aire } \\
\text { acondicionado }\end{array}$ & $\begin{array}{l}\text { Ropa con aislamiento } \\
\text { al calor o frio con } \\
\text { función protectora, } \\
\text { resistencia al rasgado } \\
\text { y alargamiento. }\end{array}$ \\
\hline & Vibración & & $\begin{array}{l}\text { Instalación de } \\
\text { plataformas o } \\
\text { sistemas } \\
\text { amortiguantes. }\end{array}$ & $\begin{array}{l}\text { Reducción del tiempo } \\
\text { de exposición y } \\
\text { pausas aplicadas. } \\
\text { Uso de guantes, } \\
\text { cinturones, plantillas } \\
\text { de calzado y } \\
\text { muñequeras } \\
\text { antivibración. }\end{array}$ \\
\hline
\end{tabular}

\section{Conclusiones}

Mediante la investigación realizada en la empresa INDUHORST en la producción de envejecedora de arroz se determinó que el personal se encuentra expuestos a muchos agentes e imprevistos que podrían generar riesgos laborales tales como RIESGOS FÍSICOS, ERGÓMICOS, MECÁNICOS, QUÍMICOS Y FUEGO Y EXPLOSIÓN. Mediante los resultados obtenidos en cuanto a los riesgos físicos, nos 
refleja un nivel muy alto en cuanto a la generación de ruidos; por otra parte en los riesgos ergonómicos los niveles se encuentras muy alto por la exposición a cargas forzadas, posturas forzadas y movimientos repetitivos; en cuanto a los riesgos mecánicos que son más frecuentes por el tipo de actividad que se genera existe un mayor riesgo en cuanto a atrapamientos por objetos, cortes en partes de su cuerpo, trabajos en altura, quemaduras por pulidora, trabajos con materiales pesados y electrocutamientos; los riesgos químicos que se frecuentan y en mayor porcentajes son producto del polvo y humo; y por último los riesgos de fuego y explosión que se encuentran expuestos es debido a la utilización de gases domésticos.

Se evaluó los procedimientos a realizar en cuanto a cada puesto de trabajo aplicando los métodos REBA y RULA, y según los indicadores se debe implementar cambios urgentes debido a malas posturas en cuanto a el área de doblado, cortado, ensamblaje y acabado; debido a la generación de ruidos se encontró una gran exposición debido a la variación de decibeles en cada una de las áreas a excepción del área de acabado. Para la lluminación se encontró que si cumple con la parte legal; con la aplicación del método Meseri para la evaluación de riesgo de incendio encontrándose en un riesgo aceptable.

Se elaboró un conjunto de procedimiento y protocolos a seguir en cada área de procesos y un plan de emergencia en el cual se establece cada uno del procedimiento a seguir referente a las diferentes situaciones que se podría presentar en la empresa, para salvaguardar la vida de las personas y personal si se llegase a presentar algún tipo de siniestros de cualquier índole, para de esta manera reducir las enfermedades y accidentes laborales en la empresa INDUHORST.

\section{Referencia}

(s.f.). Obtenido de SOLO INDUSTRIALES: https://soloindustriales.com/lasenalizacion-las-empresas/

ATEXGA PREVENCION . (s.f.). Obtenido de

http://www.atexga.com/prevencion/es/guia/riesgos-generales/senalizacionde-seguridad.php

calameo . (s.f.). Obtenido de https://es.calameo.com/read/003431364cec96cdf041b

Cavassa, C. R. (2005). Seguridad Industrial, un enfoque integral. México: Limusa, S.a. c.v.

ergonautas . (s.f.). Obtenido de

https://www.ergonautas.upv.es/metodos/niosh/niosh-ayuda.php 
Fude. (2008). Planes de seguridad e higiene laboral. Educativos. net.

Prevevisión de riesgos laborables . (09 de 11 de 2017). Obtenido de https://prevencion-riesgoslaborales.com/tipos-riesgos-laborales/

Rimac. (2014). Riesgos laborables . Obtenido de http://prevencionlaboralrimac.com/Herramientas/Matriz-riesgo

Ríos, M. G. (2002). Seguridad Laboral. Obtenido de http://www.bvsde.paho.org/bvsacd/cd46/LSI_Cap04.pdf

Tools, I. (2015). Calidad y Exelencia . Ecuador. 